\title{
SOLVENT AND STRUCTURE EFFECTS ON ELECTRONIC ABSORPTION SPECTRA OF THE ISOMERIC PYRIDINECARBOXYLIC ACIDS
}

\author{
S. Drmanić, ${ }^{\text {a* }}$ J. Nikolić, ${ }^{a}$ G. Šekularac, ${ }^{a}$ \\ B. Ranković, ${ }^{a}$ and B. Jovanović ${ }^{b}$
}

UDC 535.34:547.826/.827

Ultraviolet absorption spectra of three isomeric pyridinecarboxylic acids (picolinic, nicotinic, and isonicotinic acids) were measured in 18 various solvents, in the wavelength range from 200 to $400 \mathrm{~nm}$. In order to analyze the solvent effect on the obtained absorption maxima, the ultraviolet absorption frequencies of the electronic transitions in the carbonyl group of the examined acids were correlated using a total solvatochromic equation in the forms $v_{\max }$ $=v_{0}+s \pi^{*}+a \alpha+b \beta$ and $v_{\max }=v_{0}+s \pi^{*}+b \beta$, where $v_{\max }$ is the absorption frequency $\left(1 / v_{\max }\right), \pi^{*}$ is a measure of the solvent polarity, $\alpha$ represents a scale of the solvent hydrogen bond donor acidity, and $\beta$ represents a scale of the solvent hydrogen bond acceptor basicity. Correlation of the spectroscopic data was carried out by means of multiple linear regression analysis. The effects of two different solvent types on the ultraviolet absorption maxima of the examined acids were compared and discussed.

Keywords: ultraviolet absorption maximum, hydroxylic solvents, nonhydroxylic solvents, picolinic acid, nicotinic acid, isonicotinic acid.

Introduction. Pyridinecarboxylic acids have applications in a wide range of fields including industry, pharmacy, and biochemistry [1-4], and therefore there is interest in studying their structural and spectrochemical properties. The connection between the compound structure, solvent effect, and ultraviolet (UV) absorption spectra has been a subject of many publications [5-11]. The absorption of UV light raises electrons in the molecule to a higher energy level. Possible electronic transitions under UV light are $n \rightarrow \pi^{*}, \pi \rightarrow \pi^{*}$, and $\sigma \rightarrow \sigma^{*}[6]$. The $n \rightarrow \pi^{*}$ transition in the carboxylic group of the three isomeric pyridinecarboxylic acids (Fig. 1) dissolved in a set of solvents was analyzed in this study.

During the excitation process in the $n \rightarrow \pi^{*}$ transition, the lone electron pair from the carbonyl oxygen is promoted to an antibonding orbital which contains higher energy. The molecular structure and the solvent used can influence the wavelength of the absorption maximum. The batochromic shift signifies lower energy of the electronic $n \rightarrow \pi^{*}$ transition, and the hypsochromic shift means higher energy. The effects of solvent polarity and hydrogen bonding on absorption spectra of the examined compounds are interpreted by means of the linear solvation energy relationships (LSER) concept, developed by Kamlet and Taft [12], using a general solvatochromic equation

$$
v_{\max }=v_{0}+s \pi^{*}+a \alpha+b \beta,
$$

where $\pi^{*}, \alpha$, and $\beta$ are solvatochromic parameters; $s, a$, and $b$ are solvatochromic coefficients; the maximum absorption frequency $v_{\max }=1 / \lambda_{\max } ; v_{0}$ is the reference value, which is taken to be in the solvent cyclohexane, for which all the solvent parameters have the value zero [12]; $\pi^{*}$ is the index of the solvent dipolarity/polarizability, which is a measure of the ability of a solvent to stabilize a charge or a dipole by its own dielectric effects. The $\pi^{*}$ scale was selected to range from 0.00 for cyclohexanone to 1.00 for dimethyl sulfoxide. The $\alpha$ coefficient represents the solvent hydrogen bond donor (HBD) acidity. In other words, it describes the ability of a solvent to donate a proton in a solvent-to-solute hydrogen bond. The $\alpha$ scale extends from 0.00 for non-HBD solvents to about 1.00 for methanol. The $\beta$ coefficient is a measure of the solvent hydrogen bond acceptor (HBA) basicity and describes the ability of a solvent to accept a proton in the solute-to-solvent hydrogen bond. The $\beta$ scale was selected to extend from 0.00 for non-HBA solvents to about 1.00 for hexamethylphosphoric acid triamide.

\footnotetext{
* To whom correspondence should be addressed.
}

${ }^{\mathrm{a}}$ University of Belgrade, Karnegijeva 4, 11000 Belgrade, Serbia; e-mail: drmana@tmf.bg.ac.rs; ${ }^{\mathrm{b}}$ Institute of Chemistry, Technology and Metallurgy, University of Belgrade, Serbia. Published in Zhurnal Prikladnoi Spektroskopii, Vol. 80, No. 6, pp. 839-843, November-December, 2013. 


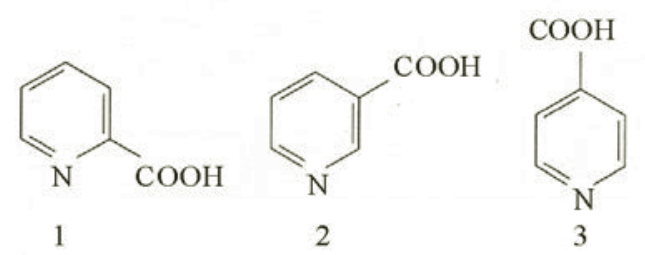

Fig. 1. Picolinic acid (1), nicotinic acid (2), isonicotinic acid (3).

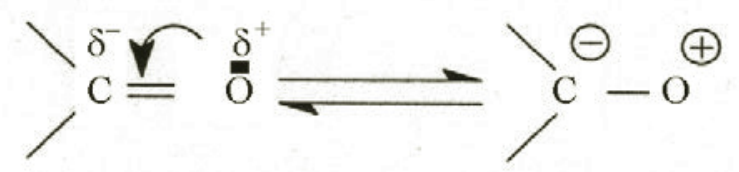

Fig. 2. The mesomeric structure in the excited state of the $n \rightarrow \pi^{*}$ transition.

Experimental. Picolinic acid, nicotinic acid, and isonicotinic acid were commercial products (Fluka — subsidiary of Sigma Aldrich Corporation, St. Louis, Missouri, USA) of p.a. quality. UV spectra of the examined compounds were recorded using a Shimadzu 1700A spectrophotometer. The wavelength range was 200-400 nm. Concentrations of the examined solutions were $10^{-4} \mathrm{~mol} / \mathrm{dm}^{3}$. The solvents used were of high purity, designed for spectroscopic measurements.

Results and Discussion. The excitation of the $n \rightarrow \pi^{*}$ transition in the carbonyl group promotes one electron from a nonbonding orbital on the oxygen to an antibonding orbital delocalized over the carbonyl group. Removal of an electron from the oxygen atom causes a decrease or even a reversal of the dipole moment, due to the mesomeric structure presence in the excited state (Fig. 2), which is opposite to the charge disposition in the ground state [8]. Such an electronic disposition can interfere with the rest of the molecule, and its stability depends on the electronic effect characteristic for the molecular structure. The absorption maxima of the examined pyridine carboxylic acids in 18 various solvents are given in Table 1.

It can be noticed that the absorption frequency values are the highest for nicotinic acid, the values for picolinic acid are in the middle, and isonicotinic acid has the lowest values. It is the other way around with the absorption wavelengths, i.e., the longest wavelengths for isonicotinic acid signify the lowest energy of the $n \rightarrow \pi^{*}$ transition. The explanation for this lies in the electronic effects in the molecules of pyridine carboxylic acids. The electron withdrawing effect of nitrogen in the ring on the carboxyl group is both inductive and resonant for isonicotinic and picolinic acids. In the case of nicotinic acid, there is only the inductive electron withdrawing effect of nitrogen due to the $m$-position of the carboxyl group. In other words, the electron withdrawing nitrogen effect is the weakest on the carboxyl group of the nicotinic acid. The conclusion can be derived (Fig. 2) that considering the reversed dipole moment and the increased electronic density around a carbon atom in the excited state, any electron withdrawing effect can stabilize the $n \rightarrow \pi^{*}$ transition of the carbonyl group. With the weaker electron withdrawing effect, the excited state of the examined electronic transition is less stable and needs more energy to be reached. The strongest electron withdrawing effects, both inductive and resonance, of the ring nitrogen is obviously on the isonicotinic acid, as it has the lowest energy of the $n \rightarrow \pi^{*}$ transition. The carboxylic group of picolinic acid, which is affected by the same resonance and inductive effects of nitrogen, seems to be somewhat less sensitive to them, as its absorption wavelengths are shorter. This is due to the formation of the hydrogen bond between nitrogen and the carboxylic proton (Fig. 3), which changes the electronic disposition in the hydroxyl group next to the carbonyl. The increased electronic density on the hydroxylic oxygen, caused by the proton being pulled away by the hydrogen bond with nitrogen, can also make the negative charge on the neighboring carbon in the excited state less stable and thereby harden the $n \rightarrow \pi^{*}$ transition.

In order to discuss the effect of solvents on the absorption spectra of the examined isomeric pyridine carboxylic acids, the absorption frequencies $\left(v_{\max }\right)$ were correlated with the Hammett-Taft solvatochromic parameters (Table 2) [13]. The obtained correlation equations in the set of hydroxylic solvents 1-9 were as follows:

picolinic acid: $\quad v_{\max }=37.40-(0.54 \pm 0.22) \pi^{*}+(0.50 \pm 0.15) \alpha+(0.61 \pm 0.35) \beta, R=0.940, s=0.07, n=9$,

nicotinic acid: $\quad v_{\max }=40.46-(4.27 \pm 0.73) \pi^{*}+(0.92 \pm 0.35) \alpha-(1.00 \pm 0.79) \beta, R=0.971, s=0.15, n=9$, 


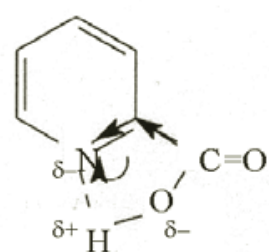

1

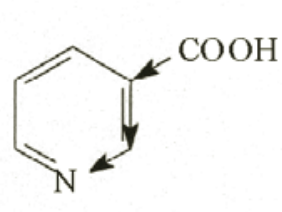

2

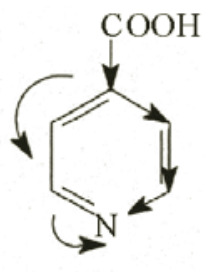

3

Fig. 3. The electron-withdrawing effects of nitrogen in the molecules of picolinic (1), nicotinic (2), and isonicotinic (3) acids, and the hydrogen bond in the molecule of picolinic acid.

TABLE 1. The Absorption Maxima $\left(v_{\max }, 10^{-3} \mathrm{~cm}^{-1}\right)$ for the Examined Pyridine Carboxylic Acids in Various Solvents

\begin{tabular}{|c|c|c|c|c|}
\hline & Solvent & Nicotinic acid & Picolinic acid & Isonicotinic acid \\
\hline 1 & Methanol & 38.3 & 37.9 & 36.8 \\
\hline 2 & Ethanol & 38.1 & 37.9 & 36.8 \\
\hline 3 & Propan-1-ol & 38.2 & 38.1 & 36.7 \\
\hline 4 & Propan-2-ol & 38.4 & 38.1 & 36.7 \\
\hline 5 & 2-Methyl-2-propanol & 38.3 & 38.1 & 36.8 \\
\hline 6 & Ethylene glycol & 37.8 & 37.7 & 36.8 \\
\hline 7 & Butan-1-ol & 38.2 & 38.1 & 36.7 \\
\hline 8 & Penta-1-ol & 38.6 & 38.2 & 36.4 \\
\hline 9 & 2-Methyl-2-butanol & 38.1 & 37.9 & 37.0 \\
\hline 10 & Acetonitrile & 38.7 & 38.1 & 36.2 \\
\hline 11 & Dimethyl sulfoxide & 37.3 & 36.9 & 36.0 \\
\hline 12 & N,N-Dimethylacetamide & 36.9 & 36.8 & 36.5 \\
\hline 13 & N-Methylpyrrolidone & 36.8 & 35.8 & 35.5 \\
\hline 14 & N-Methylformamide & 36.6 & 36.4 & 36.2 \\
\hline 15 & Tetrahydrofuran & 38.4 & 38.3 & 36.9 \\
\hline 16 & Dioxane & 38.6 & 38.5 & 36.7 \\
\hline 17 & N,N-Dimethylformamide & 37.1 & 37.0 & 36.2 \\
\hline 18 & Ethyl acetate & 38.9 & 38.7 & 37.1 \\
\hline
\end{tabular}

isonicotinic acid: $\quad v_{\max }=35.40+(3.21 \pm 0.40) \pi^{*}-(1.02 \pm 0.19) \pi^{*}+(0.65 \pm 0.44) \beta, R=0.984, s=0.08, n=9$.

In the case of the picolinic acid, solvent polarity/polarizability decreases the absorption frequency and also the energy of the $n \rightarrow \pi^{*}$ transition in the carbonyl group. Proton donor and proton acceptor solvent effects increase the absorption frequency and therefore the energy of the examined transition. Regarding nicotinic acid, both solvent polarity/polarizability and proton-acceptor solvent effect decrease the absorption frequency and therefore the energy of the $n \rightarrow \pi^{*}$ transition in the carbonyl group. Contrary to that, the proton donor solvent effects increase the energy of the same $n \rightarrow \pi^{*}$ transition. Opposite to the previous conclusion, in the case of isonicotinic acid, both solvent polarity/polarizability and proton-acceptor solvent 
TABLE 2. Solvent Parameters

\begin{tabular}{|l|l|c|c|c|c|l|c|c|c|}
\hline \multicolumn{2}{|c|}{ Solvent } & $\pi^{*}$ & $\alpha$ & $\beta$ & \multicolumn{2}{|c|}{ Solvent } & $\pi^{*}$ & $\alpha$ & $\beta$ \\
\hline 1 & Methanol & 0.60 & 0.93 & 0.62 & 10 & Acetonitrile & 0.75 & 0.19 & 0.37 \\
3 & Ethanol & 0.54 & 0.83 & 0.77 & 11 & Dimethyl sulfoxide & 1.00 & 0.00 & 0.75 \\
4 & Propan-1-ol & 0.52 & 0.78 & 0.83 & 12 & N,N-Dimethylacetamide & 0.88 & 0.00 & 0.76 \\
5 & Propan-2-ol & 0.48 & 0.76 & 0.95 & 13 & N-Methylpyrrolidone & 0.92 & 0.00 & 0.77 \\
6 & 2-Methyl-2-propanol & 0.41 & 0.68 & 1.01 & 14 & N-Methylformamide & 0.90 & 0.80 & 0.80 \\
7 & Ethylene glycol & 0.92 & 0.90 & 0.52 & 15 & Tetrahydrofuran & 0.58 & 0.00 & 0.55 \\
8 & Butan-1-ol & 0.47 & 0.79 & 0.88 & 16 & Dioxane & 0.55 & 0.37 & 0.37 \\
9 & Penta-1-ol & 0.40 & 0.84 & 0.86 & 17 & N,N-Dimethylformamide & 0.88 & 0.00 & 0.69 \\
\hline
\end{tabular}

TABLE 3. The Effect Percentage Contribution for the Hydroxylic Solvents

\begin{tabular}{|l|c|c|c|}
\hline \multicolumn{1}{|c|}{ Compound } & $\pi^{*}$ & $\alpha$ & $\beta$ \\
\hline Picolinic acid & 33 & 30 & 37 \\
Nicotinic acid & 52 & 15 & 33 \\
Isonicotinic acid & 66 & 21 & 13 \\
\hline
\end{tabular}

effect increase the absorption frequency and therefore the energy of the $n \rightarrow \pi^{*}$ transition, and the proton donor solvent effects decrease them.

As it can be seen in Table 3, the polarity/polarizability solvent effect is dominant on isonicotinic and nicotinic acids but on picolinic acid, the solvent effects are almost evenly distributed. The proton-donor effect, not dominant in any of the examined cases, has the highest contribution in picolinic acid, if it is considered separately, which can be explained by the increased electronic density around hydroxylic oxygen of the carboxylic group due to the intramolecular hydrogen bond (Fig. 3).

The correlation equations in the set of nonhydroxylic solvents 10-18 were obtained without the $\alpha$ parameter; it was excluded due to its unreliability because there were not enough data, since most applied solvents belong to the group of aprotic and have the value $\alpha=0.00$. They are presented in the equation for:

$$
\begin{array}{lll}
\text { picolinic acid: } & v_{\max }=41.36-(2.70 \pm 1.50) \pi^{*}-(3.07 \pm 1.48) \beta, R=0.939, s=0.41, n=9, \\
\text { nicotinic acid: } & v_{\max }=41.08-(1.23 \pm 1.05) \pi^{*}-(3.96 \pm 1.04) \beta, R=0.963, s=0.28, n=9, \\
\text { isonicotinic acid: } & v_{\max }=38.03-(3.19 \pm 0.64) \pi^{*}+(1.44 \pm 0.64) \beta, R=0.926, s=0.18, n=9 .
\end{array}
$$

In the case of picolinic acid, both the solvent polarity/polarizability and proton-acceptor solvent effect decrease the absorption frequency and thus the energy of the $n \rightarrow \pi^{*}$ transition in the carbonyl group. Furthermore, the nonhydroxylic solvent effect on nicotinic acid is the same: both the solvent polarity/polarizability and proton-acceptor solvent effect decrease the absorption frequency and thus the energy of the $n \rightarrow \pi^{*}$ transition in the carbonyl group. Opposite to the previous observations, in the case of the isonicotinic acid, the proton-acceptor solvent effect increases the absorption frequency and therefore the energy of the $n \rightarrow \pi^{*}$ transition in the carbonyl group, but the solvent polarity/polarizability decreases the energy of the $n \rightarrow \pi^{*}$ transition as in the other two cases. 
TABLE 4. The Effect Percentage Contribution for the Nonhydroxylic Solvents

\begin{tabular}{|l|c|c|}
\hline \multicolumn{1}{|c|}{ Compound } & $\pi^{*}$ & $\beta$ \\
\hline Picolinic acid & 47 & 53 \\
Nicotinic acid & 24 & 76 \\
Isonicotinic acid & 69 & 31 \\
\hline
\end{tabular}

TABLE 5. The Effect Percentage Contribution for the Protic Solvents

\begin{tabular}{|l|c|c|c|}
\hline \multicolumn{1}{|c|}{ Compound } & $\pi^{*}$ & $\alpha$ & $\beta$ \\
\hline Picolinic acid & 56 & 17 & 27 \\
Nicotinic acid & 72 & 4 & 24 \\
Isonicotinic acid & 73 & 9 & 18 \\
\hline
\end{tabular}

As shown in Table 4, the polarity/polarizability solvent effect is dominant in isonicotinic acid, the same as in the hydroxylic solvents. In nicotinic acid the proton-acceptor solvent effect prevails, and the effects in picolinic acid are almost evenly distributed, again as in the hydroxylic solvents.

As an addition to this study, it was interesting to observe the results in a set of solvents with various properties, both protic and aprotic, as in our previous study of the electronic absorption spectra of pyridine carboxylic acid N-oxides [11]. The most successful results were obtained with the set of 11 solvents, eight protic (methanol, ethanol, propan-1-on, propan-2-ol, ethylene glycol, 2-methyl-2-propanol, butan-1-ol, pentan-1-ol) and three aprotic (dioxane, tetrahydrofuran and ethyl acetate). The correlation equations are as follows:

$$
\begin{array}{ll}
\text { picolinic acid: } & v_{\max }=39.60-(1.36 \pm 0.38) \pi^{*}-(0.41 \pm 0.15) \alpha-(0.64 \pm 0.30) \beta, R=0.942, s=0.11, n=11, \\
\text { nicotinic acid: } & v_{\max }=41.71-(4.27 \pm 0.60) \pi^{*}-(0.22 \pm 0.21) \alpha-(1.41 \pm 0.47) \beta, R=0.962, s=0.17, n=11, \\
\text { isonicotinic acid: } & v_{\max }=34.90+(3.06 \pm 0.44) \pi^{*}-(0.40 \pm 0.17) \alpha+(0.75 \pm 0.34) \beta, R=0.952, s=0.19, n=11 .
\end{array}
$$

It can be noticed that in the given mixed set of 11 solvents the most effects are due to the decrease in the absorption frequency, and thus in the energy of the examined transition, except for the proton-acceptor solvent effect in isonicotinic acid. Such a disposition is rather similar to the nonhydroxylic solvent set examined before. The lowest values for the coefficient of the proton-donor parameter $(\alpha)$, in the case of nicotinic acid at the limit of reliability, can be explained by the choice of three aprotic solvents which all have $\alpha=0$, which decreases the parameter significance in the examined set.

As one can see in Table 5, the dominant effect in all cases is the polarity/polarizability solvent effect, followed by the proton-acceptor effect, and as expected, the proton-donor solvent effects have the smallest contribution, especially nicotinic acid. Such a distribution of the solvent effect contributions is rather uniform, unlike those for sets of the separate hydroxylic and nonhydroxylic solvents.

Conclusions. The UV absorption maxima of the three isomeric pyridine carboxylic acids were determined in 18 various solvents. The observed electronic transition was the $n \rightarrow \pi^{*}$ in the carbonyl group of the carboxylic acids. The absorption maxima, expressed as UV absorption frequencies, had different values depending on the solvent or compound structure. They were correlated by the Hammett-Taft total solvatochromic equation, separately for hydroxylic and nonhydroxylic solvents, and also in the set of the chosen 11 solvents of various properties. The domination, contributions, and the directions of solvent effects on the absorption frequencies, and therefore on the energy of the examined transition, varied with the solvent set, displaying the important role that the solvent choice plays in the UV spectroscopic analysis of organic compounds.

Acknowledgements. The authors are grateful to the Ministry of Education and Science of The Republic of Serbia for financial support (Project 172013). 


\section{REFERENCES}

1. J. Singh, P. K. Dutta, J. Macromol. Sci. A: Pure Appl. Chem., 48, 246 (2011).

2. S. Funasaka, T. Mukaiyama, Bull. Chem. Soc. Jpn., 81, 148 (2008).

3. A. Zahradnikova, I. Minarovic, I. Zahradnik, J. Pharmacol. Exp. Ther., 322, 638 (2007).

4. N. Haginoya, S. Komoriya, K. Osanai, T. Yoshino, T. Nagata, M. Nagamochi, R. Muto, M. Yamaguchi, T. Nagahara, H. Kanno, Heterocycles, 63, 1555 (2004).

5. A. T. Nielsen, J. Org. Chem., 22, 1539 (1957).

6. C. N. R.Rave, Ultraviolet and Visible Spectroscopy: Chemical Applications, 2nd ed., Butterworks, London, 109-115 (1967).

7. J. N. Gardner, A. R. Katritzky, J. Chem. Soc., 4375 (1975).

8. J. B. Nikolić, G. S. Ušćumlić, V. Krstić, J. Serb. Chem. Soc., 65, 353 (2000).

9. G. S. Ušćumlić, A. A. Kshad, D. Ž. Mijin, J. Serb. Chem. Soc., 68, 699 (2003).

10. D. Ž. Mijin, G. S. Ušćumlić, N. U. Perišić-Janjić, N. V. Valentić, Chem. Phys. Lett., 418, 223 (2006).

11. S. Ž. Drmanić, J. B. Nikolić, A. D. Marinković, G. M. Šekularac, B. Ž. Jovanović, CICEQ (2012) doi:10.2298/ CICEQ120326073D (article published online).

12. M. Kamlet, J. Abboud, R.W. Taft, Prog. Phys. Org. Chem., 13, 485 (1983).

13. A. F. Lagalante, R. J. Jacobson, T. J. Bruno, J. Org. Chem., 61, 6404 (1996). 